\title{
From molecules to particles in silane plasmas
}

\author{
A. A. Howling, C. Courteille, J.-L. Dorier, L. Sansonnens, and Ch. Hollenstein \\ Centre de Recherches en Physique des Plasmas, Ecole Polytechnique Fédérale \\ Lausanne, Avenue des Bains 21, CH-1007 Lausanne, Switzerland
}

\begin{abstract}
Particle formation has been investigated experimentally from the initial molecular precursors up to the final micron-sized particles in a low pressure silane if capacitive discharge. Neutrals and ions were studied by quadrupole mass spectrometry in power-modulated plasmas: Whole series of negative ions were observed, ranging from monosilicon anions through to nanometric clusters. Anion confinement results in particles and conversely, anion de-trapping can inhibit particle formation. Plasma polymerisation is considered in terms of neutral and ionic species. Laser light scattering measurements show that particles appear during a rapid coalescence phase and possible mechanisms are discussed.
\end{abstract}

\section{INTRODUCTION}

Particles during silane plasma processing are a source of contamination [1], all the more serious as device areas increase and feature sizes diminish. Despite clean room precautions, however, particles may still ultimately form during the process itself by homogeneous gas-phase nucleation inherent in the plasma chemistry. This is an example of plasma state polymerisation which limits the maximum process rate for particle-free deposition [2]. If formed, micrometric particles are negatively-charged by electron-ion flux equilibration at their surface [3-5] whereupon they are trapped by the plasma sheath potentials and accumulate in electrostatic suspension between the electrodes.

It is obvious that macromolecular intermediates must have time to form and grow by successive reactions in the plasma. To eliminate particles, therefore, it suffices to prevent the formation of these particle precursors. The precursors themselves may be neutral or ionic (positive or negative), and the growth reactions may also involve neutrals and/or ions. Different plasma conditions for a given process may favor different polymerisation pathways [6].

In low power, low pressure silane plasmas, the appearance of particles after plasma ignition can take many seconds to several minutes $[7,8]$. Negative ions are retained by the plasma sheaths, which suggests that plasma polymerisation might proceed via an anionic pathway $[3,6,9-12]$. In this paper, we use quadrupole mass spectrometry of neutrals, cations and anions in a power-modulated rf silane plasma [9] to assess their rôle in particle formation. ON/OFF rf power modulation is necessary to allow the sheath potentials to collapse so that negative ions can diffuse to the electrodes for detection $[9,13]$.

\section{EXPERIMENTAL METHOD}

The experimental apparatus [9] is a conventional parallel-plate of capacitive reactor comprising two 13-cmdiam electrodes with a $2.5 \mathrm{~cm}$ electrode gap. The gas inlet, in the side wall of the grounded vacuum chamber, was a $30 \mathrm{sccm}$ flow of pure silane at 0.1 mbar reactor pressure. The if power $(\sim 0.03 \mathrm{~W} \mathrm{~cm}-3)$ was ON/OFF modulated at $\mathrm{kHz}$ frequencies with an excitation frequency of $10-30 \mathrm{MHz}$ capacitivelycoupled to the rf electrode via a $\pi$ matching network. The specific plasma parameters are given in each figure caption. Polarisation-sensitive Mie scattering of a laser was used for quantitative $[7,14]$ estimations of particle size and number density during powder formation in the plasma.

Two different quadrupole mass spectrometers were separately employed, both equipped for neutral analysis, ion extraction, mass and energy measurements. Single-stage differential pumping maintained a spectrometer pressure of $5.10^{-6} \mathrm{mbar}$ for $0.1 \mathrm{mbar}$ of silane in the reactor. The Hiden Analytical Limited Plasma Monitor type HAL-EQP 500 measured negative ions up to the limit of $512 \mathrm{amu}$ when mounted either in the ground electrode or to the side of the electrode gap [9]. Ions entered the monitor first through a 5 -mm-diam orifice in the grounded spectrometer head and then a $0.1 \mathrm{~mm}$ aperture in the biased extractor electrode; $a+40 \mathrm{~V}(-50 \mathrm{~V})$ bias was used for negative (positive) ion sampling. A $45 \mathrm{deg}$. sector electrostatic 
analyser selected the transmitted ion energy before the quadrupole mass filter and channeltron detector. The Balzers Plasma Process Monitor type PPM 421, with mass range option up to $2048 \mathrm{amu}$, was positioned to the side of the electrode gap. Ions enter the extractor head through a $0.1 \mathrm{~mm}$ orifice exposed to the plasma and a positive extractor bias of a few volts was used for negative ion sampling. The transmitted ion energy here was selected by a cylindrical mirror analyser. The important question of non-perturbative anionic cluster sampling is addressed after the following section; however, we note here the practical difficulty of mass spectrometry in deposition plasmas where powder particles or deposited film can block the sampling aperture $[9,10,13]$. Depending on the plasma parameters, the monitor extraction head was cleaned in acid after every few hours of plasma exposure.

\section{RESULTS AND DISCUSSION}

Ion Mass Spectra in Power-Modulated Silane Plasmas

In continuous if plasmas, negative ions are retained by the sheath potentials and mass spectrometry can only measure neutrals and positive ions. During the afterglow period of an ON/OFF rf-power-modulated silane plasma, the electron density decays in $30-50 \mu \mathrm{s}[9,13]$, the sheath potentials collapse and negative ions can then escape. Their density decays away completely unless the plasma is reignited whereupon the remaining negative ions are re-trapped [9]. Mass spectrometry can therefore measure negative ions provided that the modulation frequency is below $10 \mathrm{kHz}$ so that the sheath has time to collapse $[9,13,15]$. The different types of negative ion diffuse from $\mathrm{kHz}$-modulated plasmas with the same low energy [9] $(<1$ $\mathrm{eV}$ ) as measured by both mass spectrometers. This energy can reach a few $\mathrm{eV}$ in longer afterglows (several milliseconds), probably because a reversed sheath voltage develops on this timescale.

Figure 1(a) shows the negative ion mass spectrum [16] obtained in a low power, low pressure silane plasma. The mass resolution was strongly degraded to maximise transmission and each peak represents the combined intensities of all ions having the same number of silicon atoms, irrespective of their hydrogen content. A complete sequence extends from monosilicon hydride anions through to mass $1300 \mathrm{amu}$; the latter correspond to nanometric anionic clusters containing 44 silicon atoms. This is much heavier than the observed positive or neutral counterparts (highest mass $\sim 200 \mathrm{amu}$ ) for these plasma parameters $[9,17,18]$. By analogy with carbanions (carb. + anion), the polysilicon hydride anions are here defined as silanions.
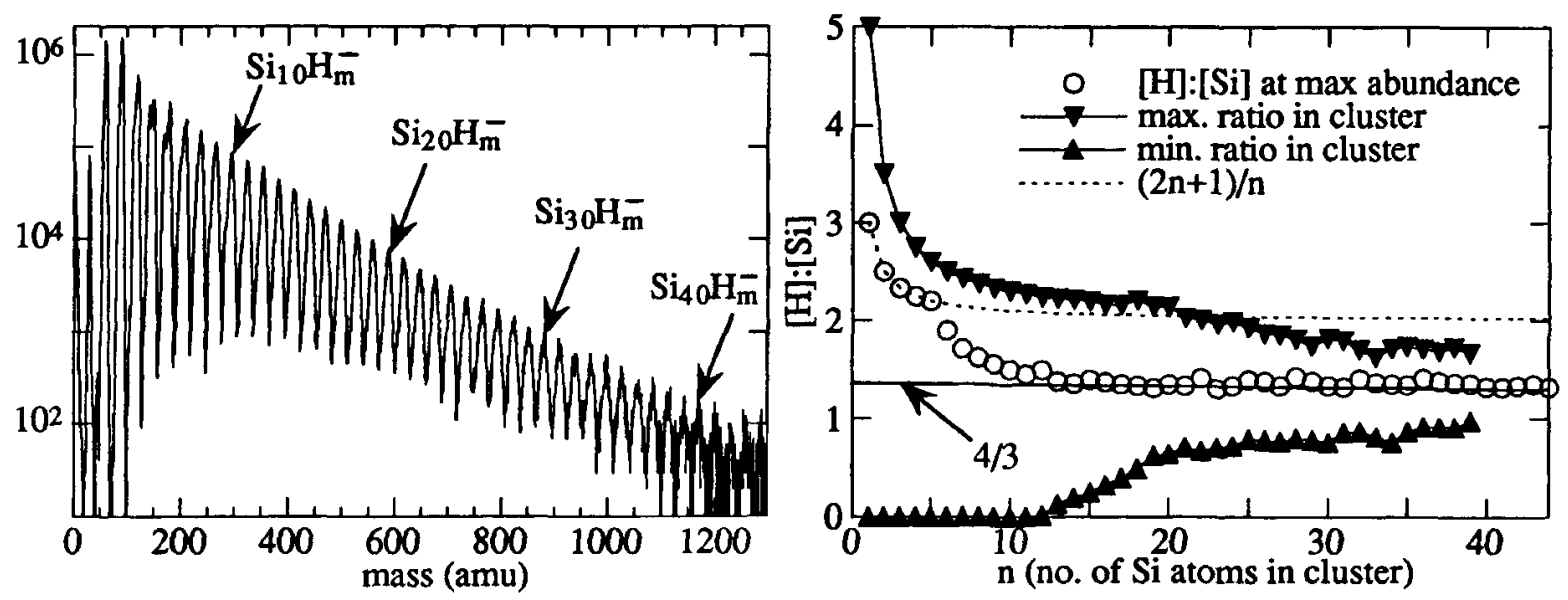

Fig. 1(a) Raw data in counts/s with the Balzers monitor. Fig. 1(b) Ratio of $\mathrm{H}$ to $\mathrm{Si}$ atoms of anions in (a). 0.1 mbar silane, electrodes at $200^{\circ} \mathrm{C} .30 \mathrm{MHz}, 8 \mathrm{~W}$ average power ON/OFF at $1 \mathrm{kHz}$ and $30 \%$ duty cycle.

These anionic clusters are stable because they survive for hundreds of microseconds during transit in the mass spectrometer [9]. Atomic silicon has a positive electron affinity $(1.385 \mathrm{eV}, \sim 40 \%$ of fluorine and chlorine) as do silane radicals $[11,12]$, and the general trend is that the electron affinity increases with cluster size towards the work function of silicon at $4.85 \mathrm{eV}$ [12]. Intermediate peaks are absent in the anion mass spectrum which shows that the clusters are singly-attached, since doubly-charged clusters with an odd number of silicon atoms would have half-integral charge-to-mass ratios. This is to be expected because Coulomb repulsion means that attachment of the second electron is likely to be endothermic. In contrast to single electron attachment, multiple electron charging of a macroscopic particle in a plasma is due to a dynamic equilibrium of electron and ion flux at its surface; these electrons disappear when the particle is no longer immersed in plasma. This is a different phenomenon from attachment and is not expected to occur before the cluster reaches several $\mathrm{nm}$ in size [3-5]. 

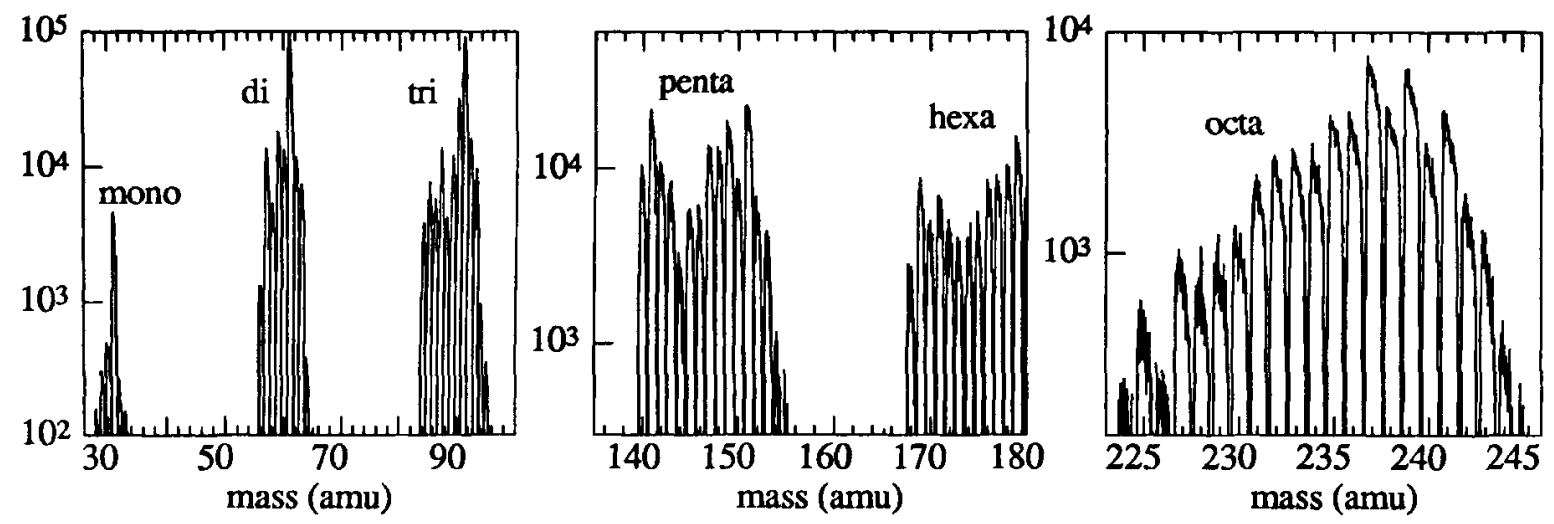

Fig. 2(a),(b),(c) Raw data in counts/s using the Balzers spectrometer, showing the individual anions within several of the broad peaks in Fig. 1(a) for the same plasma parameters.

Figure 2 shows silanion mass spectra with resolution of the individual $\mathrm{Si}_{\mathrm{n}} \mathrm{H}_{\mathrm{m}}^{-}$within several of the broad peaks of Fig. 1(a). For a given silanion group containing $n$ silicon atoms (up to $n \sim 10$ ), the anions range from $\mathrm{Si}_{n}^{-}$through to $\mathrm{Si}_{n} \mathrm{H}_{2 \mathrm{n}}+3$ [15] (anions with one or two extra atomic mass units are attributed to the minority isotopes of silicon). Anions with $n \leq 4$ (Fig. 2(a)) present a clear maximum signal for $\mathrm{Si}_{n} \mathrm{H}_{2 n+1}$ which is isoelectronic with its equivalent saturated neutral $\mathrm{Si}_{n} \mathrm{H}_{2 n+2}$ [15]. Anions with five silicon atoms (to a lesser extent, 6 silicon atoms) have particularly-abundant pure silicon clusters $\mathrm{Si} \overline{5}$ as well as $\mathrm{Si}_{5} \mathrm{H}^{-}$-Heavier clusters, such $\mathrm{as} \mathrm{Si}_{8} \mathrm{H}_{\mathrm{m}}^{-}$in $\mathrm{Fig}$. 2(c), exhibit a broad intensity distribution with respect to $\mathrm{H}$-content. This may be because energy constraints are relaxed for large clusters having many internal degrees of freedom.

Figure 1(b) plots the ratio of $\mathrm{H}$ atoms to $\mathrm{Si}$ atoms within each anionic cluster of Fig. 1(a): There is a transition in the maximum-abundance $[\mathrm{H}]$ :[Si] ratio at $n \sim 5$, passing from $\mathrm{Si}_{n} \mathrm{H}_{2 \mathrm{n}+1}$ dominance to a sizeindependent ratio $[\mathrm{H}]:[\mathrm{Si}] \sim 4 / 3$ for cluster sizes above $n \sim 10$. If the $\mathrm{Si}_{n} \mathrm{H}_{2 \mathrm{n}+1}^{-1}$ sequence were followed indefinitely, the ratio would have been 2 in the limit of large $n$ (Fig. 1(b)). This shows that silanions are highly cross-linked, three-dimensional structures [19]. Given the absence of double bonds in silicon clusters and neglecting dangling bonds, $[\mathrm{H}]:[\mathrm{Si}]=4 / 3$ corresponds to equal numbers of $\mathrm{Si}-\mathrm{H}$ and $\mathrm{Si}-\mathrm{Si}$ bonds within the dominant clusters. The constant ratio with cluster size shows that the clusters are not pure $\mathrm{Si}_{\mathrm{n}}$ cores covered with surface $\mathrm{H}$ atoms; neither are they 'cluster ions' in the strict sense of the term (of type $\mathrm{SiH}_{\mathrm{m}}^{-} \cdot\left(\mathrm{SiH}_{4}\right)_{\mathrm{n}}$, for which [H]:[Si] $\left.\rightarrow 4\right)$.

\section{Ion Sampling in Modulated Plasmas for Anionic Cluster Sources}

It is important to check whether the negatively-charged clusters in Figs. 1 and 2 are properties of the plasma itself and not merely artefacts of the sampling method. Many sources use gas jet expansion through similar size orifices to create clusters by adiabatic cooling but these employ pressures several orders of magnitude higher. High Pressure Ion Mass Spectroscopy, as defined in Ref. 20, at 10 mbar with a 0.05-mm-diam orifice has been proven to be free from adiabatic clustering. For our case at 0.1 mbar, with a neutral mean free path of $\sim 1 \mathrm{~mm}$ and a $0.1 \mathrm{~mm}$-diam orifice, the nozzle Knudsen number is of order 10 corresponding to collisionless molecular flow (effusion) into the mass spectrometer. The estimated fraction of ions reaching the detector without collision after entering the orifice is $\exp \left(-\pi \sigma R n_{d} / 4\right)[21] \sim 95 \%$ (taking ionneutral cross-section $\sigma \sim 5 \times 10^{-15} \mathrm{~cm}^{2}$, orifice radius $\mathrm{R}=5 \times 10^{-3} \mathrm{~cm}$, neutral density at entrance $\mathrm{n}_{\mathrm{o}}=$ $2.4 \times 10^{15} \mathrm{~cm}^{-3}$ ). In addition, the energy selector in both spectrometers means that any ion undergoing inelastic collisions such as clustering after entering the extraction orifice would appear to have negative energy - this is not observed. Although the ion extraction geometry and overall design of the two mass spectrometers used are different, the measured energy distributions and abundances $[9,15]$ are nevertheless the same. Finally, time-resolved experiments also show that the anions were formed during the plasma [13] and not by attachment to heavy neutrals [9]. Taken together with other measurements [10,22] and numerical simulations $[2,23]$ of significant negative ion densities in silane plasmas, we conclude that Figs. 1 and 2 represent anions produced in the plasma and are not instrumental artefacts.

Modulated plasmas of electronegative gases could be exploited as volume sources of anionic clusters: The plasma provides a reservoir of high energy electrons, radicals and rovibrationally-excited molecules with potential for producing cluster series which might otherwise be energetically inaccessible. The anionic clusters are also collisionally-thermalised (so avoiding evaporation from internally-hot clusters in supersonic jets) without necessitating high pressures [20] because of their arbitrarily-long trapping time in the gas. Furthermore, charged clusters facilitate mass selection in spectrometers. The stability of the silanions and their immunity to detachment in the spectrometer electric fields could be exploited for time-offlight mass spectrometry and semiconductor cluster studies. 


\section{Anion Polymerisation}

From Fig. 2(a), $\mathrm{SiH}_{3}^{-}$has by far the highest density of the monosilicon hydride anions in this type of discharge $[11,12,15]$. It is most likely produced by dissociative attachment on silane and/or by attachment to silane radicals [11]; these rates may be enhanced for the excited states [24] which exist in plasmas. The abundance ratio of consecutive anion groups is an order of magnitude higher than for neutrals [9] and so the higher-mass anions cannot be attributed to attachment to higher-order silane neutrals (see Ref. 9 for additional arguments). The implication is that silanions are formed in a polymerisation sequence. Silane addition with $\mathrm{H}_{2}$ elimination [12] starting from $\mathrm{SiH}_{3}^{-}$would explain the dominance of $\mathrm{Si}_{n} \mathrm{H}_{2}^{-} \mathrm{n}+1$ anions as observed in the early sequences of Fig. 2 . However, $\mathrm{SiH}_{3} \overline{3}$ is very slow to cluster with ground-state silane [11]. Experimental observations therefore suggest that plasma-created products are involved in anion growth [24], ie that the anion polymerisation proceeds via plasma-activated monomers. This would be experimentally difficult to study for isolated reactions with very low pressure techniques since neither participant is a ground state neutral. Possible plasma reactants with anions are: i) excited (electronic or vibrational) silane [24]; ii) silane radicals [11,12,24]; and/or iii) anion-cation association [11,12] followed by attachment. Further experimental work is required to clarify this situation, including the influence of gas temperature [12]. The low [H]:[Si] ratio of $4 / 3$ in Fig. 1(b) shows that cluster growth involves chemical reactions with silane and hydrogen elimination [12], and/or with $\mathrm{H}$-depleted radicals or cations. The exponential decay of the mass spectrum in Fig. 1(a) is typical of weak clustering conditions where clusters are predominantly formed by monomer addition and cluster-cluster aggregation is rare (slow flocculation); the latter condition is guaranteed since inelastic anion-anion collisions are prohibited by Coulomb repulsion.

\section{Experimental Evidence for Negative Ions as Powder Precursors in Low Pressure Silane Plasmas}

Particle formation in $\mathrm{rf}$ silane plasmas is strongly reduced by square-wave power modulation at $\mathrm{kHz}$ frequencies $[10,15,25]$. In previous work $[7,9,15]$ with plasma conditions suitable for high quality amorphous silicon deposition, we demonstrated that powder does not form when anions are observed to escape (modulation frequency $<3 \mathrm{kHz}$ ), and that powder does form when the anions do not have time to be evacuated in the afterglow (modulation frequency $>3 \mathrm{kHz}$ ). This was summarised as an anti-correlation between the observation of anions escaping the plasma and the observation of powder particles forming in the plasma [15]. A physical explanation for the suppression of highly-polymerised anions near $1 \mathrm{kHz}$ modulation frequency is that the plasma period is then too short for the anions to reach high masses before the reservoir of low mass anions is evacuated in each subsequent afterglow period [7,9].

Supplementary evidence was obtained by a partial-depth modulation experiment, based on an observation by Overzet $e t$ al [13] that the sheath does not collapse and anions cannot escape unless the modulation depth of the if voltage exceeds $\sim 85 \%$. The results of our experiment are as follows: In Fig. 3 (a) with ON/OFF power modulation (corresponding to $100 \%$ modulation depth), a negative ion flux was measured during the afterglow but no powder was detectable - this is the usual situation as described above $[7,9,15]$. After more than 4.5 minutes without powder formation, the rf voltage during the afterglow was set at $48 \mathrm{~V}$ (Fig. 3(b)), whereupon the sheath no longer fully collapsed: The anion flux abruptly disappeared and powder appeared after $40 \mathrm{~s}$. The principle of the experiment is that the magnitudes and dynamics of neutral species production and loss rates are essentially unchanged by going from Fig.3 (a) to (b) since neutrals are not affected by the sheaths, whereas the anion flux is switched on or off. Indeed, the

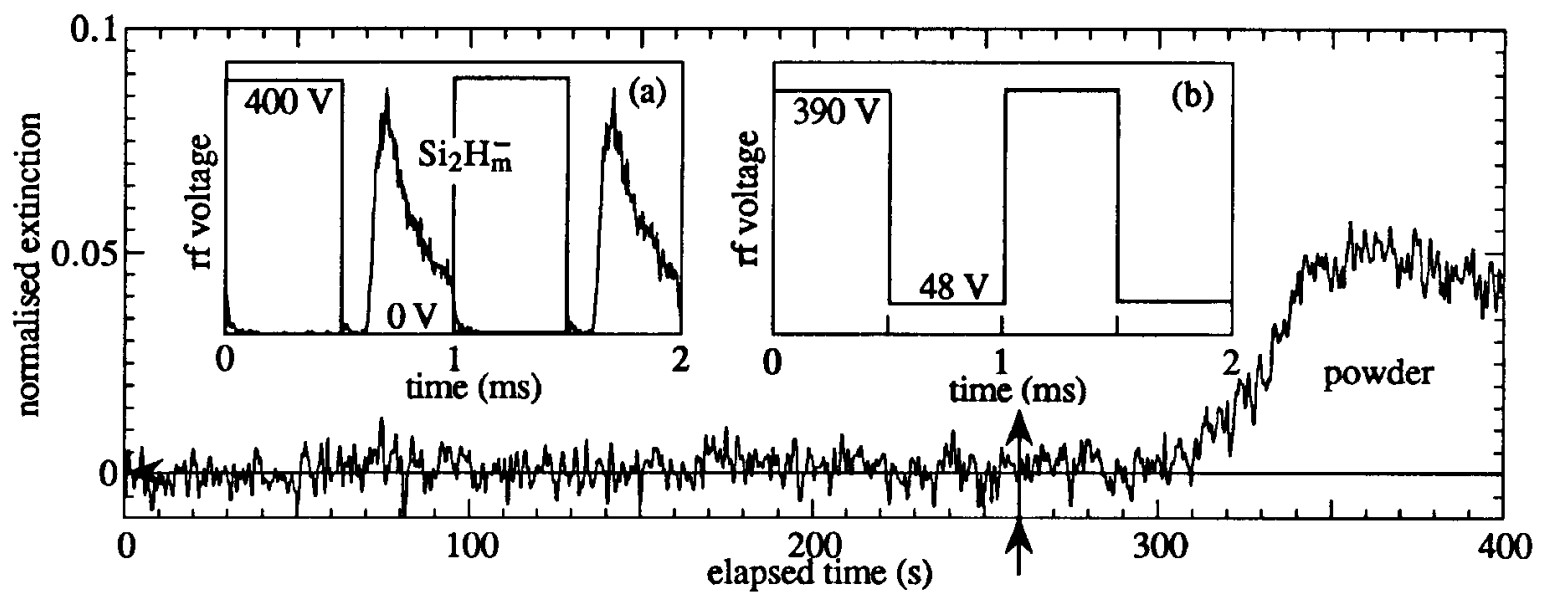

Fig. 3. Partial-depth modulation experiment. Powder was monitored by the extinction of a He-Ne laser beam. The disilicon anion flux [9] in (a) was measured with the Hiden monitor and a multichannel scalar.The initial rf voltage amplitude modulation is shown in inset (a). Arrows mark the time when the afterglow voltage was increased to $48 \mathrm{~V}$ (inset (b)). Electrode temperature $66^{\circ} \mathrm{C}, 0.1$ mbar silane and $10 \mathrm{MHz}$ rf frequency; the time-averaged if power for both (a) and (b) was $10 \mathrm{~W}$. 
neutral mass spectrum was invariant in form and intensity throughout the experiment and the measured disilane and trisilane neutral fluxes showed no variation over the modulation cycle (the measured 1/e decay time for these neutral fluxes after plasma extinction was $\sim 0.1 \mathrm{~s}$ ). The partial-depth modulation technique therefore discriminates between neutrals and anions (note that the ON/OFF durations must be chosen according to the plasma parameters in conjunction with anion measurements: If the OFF period is too short to allow significant anion diffusion, or the $\mathrm{ON}$ period so long as to result in strong polymerisation during a single plasma period, then the results are meaningless when interpreted in terms of anions). Our conclusion is that negative ions are the precursors to particle growth in these low pressure plasmas.

\section{A Particle Formation Paradox}

For weak clustering, it is important to distinguish between the 'polymerisation pathway', which is the growing cluster (particle precursor) species, and the reactive 'monomer' species, which enable the cluster to grow by repeated addition reactions - both are necessary for particle formation but are not necessarily the same species (negative/positive/neutral). If neutrals were the pathway species in low pressure experiments in which particles form slowly in a continuous plasma, the neutral dwell time would have to be at least a second. But $\mathrm{kHz}$ modulation is observed to eliminate powder formation, imposing a neutral precursor lifetime of less than a millisecond (NB it is not sufficient that the reactive monomer species disappear rapidly [25] because if the pathway species remains for further growth in subsequent plasma periods, the powder would still continue to grow albeit more slowly due to a lower monomer density). This is a paradoxical situation for neutrals which can only be resolved by taking negative species to be the polymerisation pathway - their dwell time in continuous plasmas is indefinitely long due to sheath trapping, and their lifetime is short in modulated plasmas due to expulsion by reversed sheath fields. Moreover, the submillisecond timescale for expulsion implies a low inertia negative species, ie molecular anions, and not submicron particles charged negatively by the plasma.

\section{Neutral and Cation Cluster Formation}

Other pathways may predominate in high power $\left(\sim 0.8 \mathrm{~W} \mathrm{~cm}^{-3}\right)$, high pressure $(\sim 1 \mathrm{mbar})$ silane plasmas in which particle formation occurs in a fraction of a second $[25,26]$. Mandich and Reents [18] disqualify positive ions since bottlenecks occur in cation nucleation (although exceptions exist $[19,27]$ ) and furthermore they are rapidly lost by evacuation across the plasma sheaths. This leaves the possibility of a rapid neutral pathway where $\mathrm{SiH}_{2}$ insertion into higher silanes is proposed $[25,26]$. If neutral clusters can reach critical size [6] or become large enough (thousands of atoms) to be negatively charged by the rf plasma $[4,5]$ before being lost from the discharge, there is no need to invoke a slower, parallel anionic pathway. The strong fall-off with mass in the neutral mass spectrum $[9,17]$ could be the low-mass portion of a bimodal mass distribution typical of strong clustering in which cluster-cluster aggregation dominates (rapid flocculation). If an absence of significant polymerisation is an empirical condition for 'device quality' deposition [2], then rapid neutral polymerisation is in any case far from these conditions. Starting with powder-free deposition, gradually increasing the deposition rate would probably lead first of all to slow cluster growth via anions. An alternative possibility is that continual attachment/detachment cycling could be responsible for extending the cluster average lifetime [6]. Nevertheless, in all of our low pressure experiments, the mass spectrum of neutrals was invariant with or without powder formation.

\section{From Clusters to Particles}

The appearance of powder is associated with a rapid coalescence phase in which nanometric clusters [8] agglomerate to form larger, monodisperse particles $\left(10^{2} \mathrm{~nm}\right)[7,8,14,28]$ - see Fig. 4 at 20 seconds. This

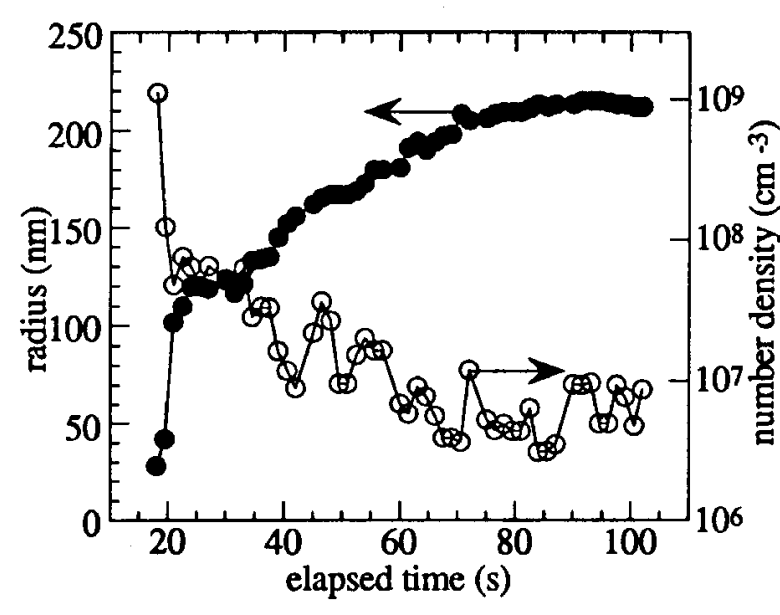

Fig. 4 Evolution of particle size and number density by polarization-sensitive Mie scattering [14].

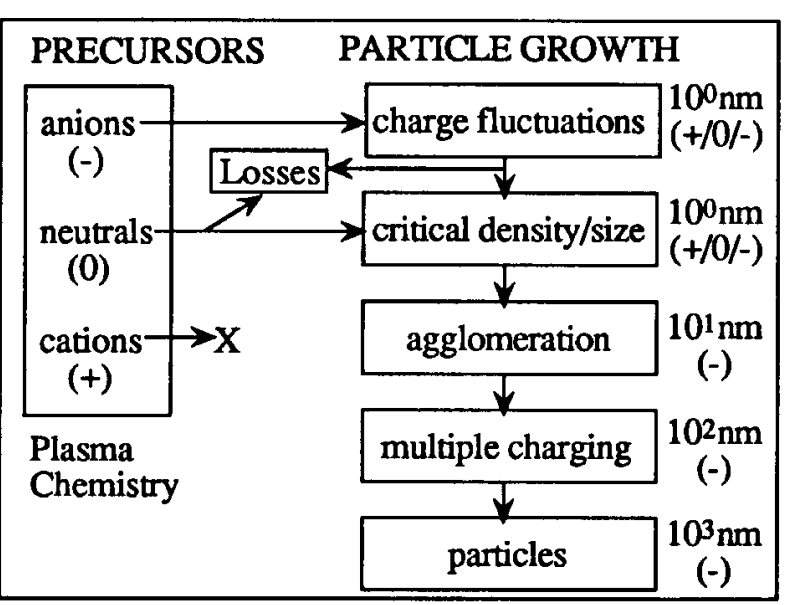

Fig. 5 Tentative scheme for particle growth from molecules in low pressure silane plasmas. 
onset of cluster-cluster aggregation can be described by free cluster nucleation theory assuming a critical size of a few $\mathrm{nm}$ and an initial cluster number density of $109-11 \mathrm{~cm}^{-3}$. Aggregation of anionic clusters is excluded until they become large enough to experience $+/$ - charge fluctuations due to statistical variations in electron/ion fluxes to their surface $[4,5]$. Once coalescence has occurred, all particles of a few tens of $\mathrm{nm}$ size will rapidly acquire a multiple electronic charge thus preventing further mutual aggregation. Subsequent growth would be via accretion of neutrals and cations in processes similar to film growth [12]. This description is summarised in Fig. 5.

\section{CONCLUSIONS}

Neutral and ionic molecules have been studied in low pressure, low power silane if plasmas using mass spectrometry to assess their rôles in particle formation. Complete sequences of stable, singly-attached negative ions were observed from monosilicon anions through to nanometric anionic clusters. Partial-depth power modulation experiments show that the anionic flux can be switched on or off and that this controls the formation of powder. We infer that anions are the initial precursors of particles for these plasma conditions. Anion polymerisation probably involves plasma-activated products: Further experiments are necessary to identify these reactions and the coalescence mechanism which results in particle formation.

Acknowledgments

We thank Drs. Rettinghaus and Korner of Balzers AG, and P. Hatton and colleagues of Hiden Analytical Limited. This work was funded by Swiss Federal Research Grants BBW 93.0136 (for Brite-Euram project BE-7328) and BEW 9400051.

\section{REFERENCES}

1. G. S. Selwyn, Plasma Sources Sci. Technol. 3, 340 (1994).

2. M. J. Kushner, J. Appl. Phys. 63, 2532 (1988).

3. A. Garscadden, B.N. Ganguly, P. D. Haaland, and J. Williams, Plasma Sources Sci. Technol. 3, 239 (1994).

4. A. C. Breslin and K. G. Emeleus, Int. J. Electronics 31, 189 (1971).

5. J. Goree, Plasma Sources Sci. Technol. 3, 400 (1994).

6. S. J. Choi and M. J. Kushner, J. Appl. Phys. 74, 853 (1993).

7. Ch. Hollenstein, J.-L. Dorier, J. Dutta, L. Sansonnens, and A. A. Howling, Plasma Sources Sci. Technol. 3, 278 (1994).

8. L. Boufendi, J. Hermann, A. Bouchoule, B. Dubreuil, E. Stoffels, W. W. Stoffels, and M. L. de Giorgi, J. Appl. Phys. 76, 148 (1994).

9. A. A. Howling, L. Sansonnens, J.-L. Dorier, and Ch. Hollenstein, J. Appl. Phys. 75, 1340 (1994).

10. J. T. Verdeyen, J. Beberman, and L. Overzet, J. Vac. Sci. Technol. A 8, 1851 (1990).

11. P. Haaland, J. Chem. Phys. 93, 4066 (1990).

12. J. Perrin, Ch. Böhm, R. Etemadi and A. Lloret, Plasma Sources Sci. Technol. 3, 252 (1994).

13. L. J. Overzet, J. H. Beberman and J. T. Verdeyen, J. Appl. Phys. 66, 1622 (1989).

14. J.-L. Dorier, Ch. Hollenstein, and A. A. Howling, J. Vac. Sci. Technol. A. 13, 918 (1995).

15. A. A. Howling, J.-L. Dorier, and Ch. Hollenstein, Appl. Phys. Lett. 62, 1341 (1993).

16. C. Courteille, L. Sansonnens, J. Dutta, J.-L. Dorier, Ch. Hollenstein, A. A. Howling, and U. Kroll, Proc. 12th Eur. Photovoltaic Solar Energy Conf., Amsterdam, 1994, p. 319.

17. I. Haller, Appl. Phys. Lett. 37, 282 (1980).

18. M. L. Mandich, W. D. Reents, and K. D. Kolenbrander, Pure \& Appl. Chem. 62, 1653 (1990).

19. T. P. Martin and H. Schaber, J. Chem. Phys. 83, 855 (1985).

20. A. W. Castleman, P. M. Holland, D. M. Lindsay, and K. I. Peterson, J. Am. Chem. Soc. 100, 6039 (1978).

21. J. Liu, G. L. Huppert, and H. H. Sawin, J. Appl. Phys. 683916 (1990).

22. M. Shiratani, T. Fukuzawa, K. Eto and Y. Watanabe, Jpn. J. Appl. Phys. 31, L1791 (1992).

23. J.-P. Boeuf, Phys. Rev. A 36, 2782 (1987).

24. L. A. Pinnaduwage, M. Z. Martin, and L. G. Christophorou, Appl. Phys. Lett. 65, 2571 (1994).

25. Y. Watanabe, M. Shiratani and H. Makino, Appl. Phys. Lett. 57, 1616 (1990).

26. S. Veprek, K. Schopper, O. Ambacher, W. Rieger and M. G. J. Veprek-Heijman, J. Electrochem. Soc. 140, 1935 (1993).

27. M. L. Mandich and W. D. Reents, J. Chem. Phys. 96, 4233 (1992).

28. L. Boufendi, A. Plain, J. Ph. Blondeau, A. Bouchoule, C. Laure, and M. Toogood, Appl. Phys. Lett. 60, 169 (1992). 